\title{
Stratification polyphonique dans les musiques d'Asie du sud-est
}

\section{Ki Mantle Hood}

Traducteur : Isabelle Schulte-Tenckhoff

\section{OpenEdition \\ Journals}

Édition électronique

URL : http://journals.openedition.org/ethnomusicologie/1375

ISSN : 2235-7688

Éditeur

ADEM - Ateliers d'ethnomusicologie

Édition imprimée

Date de publication : 31 octobre 1993

Pagination : $3-10$

ISBN : 2-8257-0485-7

ISSN : 1662-372X

Référence électronique

Ki Mantle Hood, «Stratification polyphonique dans les musiques d'Asie du sud-est », Cahiers

d'ethnomusicologie [En ligne], 6 | 1993, mis en ligne le 02 janvier 2012, consulté le 10 décembre 2020.

URL : http://journals.openedition.org/ethnomusicologie/1375

Ce document a été généré automatiquement le 10 décembre 2020.

Tous droits réservés 


\title{
Stratification polyphonique dans les musiques d'Asie du sud-est
}

\author{
Ki Mantle Hood \\ Traduction : Isabelle Schulte-Tenckhoff
}

\section{NOTE DE L'ÉDITEUR}

Traduit de l'anglais

1 Les bruits stridents du Nouvel An chinois sont polyphoniques au sens propre. Bien sûr, personne n'a jamais voulu leur appliquer le terme de polyphonie. Par ailleurs, en cette époque d'intérêt universel pour les théories musicales, ce terme ne devrait pas se limiter à la musique européenne, qu'il s'agisse de la musique modale du Moyen Âge et de la Renaissance ou de la musique tonale du baroque et des époques suivantes. Du point de vue culturel, il n'est peut-être même pas viable, puisque les compositeurs d'avant-garde du XX $\mathrm{X}^{\mathrm{e}}$ siècle ne l'utilisent apparemment pas, quel que soit le degré de proximité de leur musique par rapport à l'univers sonore du Nouvel An chinois.

Avant que ce terme ne soit définitivement écarté en raison de sa trop forte connotation culturelle, j'aimerais élucider la notion de stratification polyphonique par référence à la musique du gamelan javanais, à d'autres traditions musicales d'Asie du sud-est, ainsi qu'à la conception générale de la polyphonie. Précisons qu'il s'agit bien ici de stratification polyphonique et non de polyphonie stratifiée. La distinction est essentielle.

Dans un contexte différent de la géologie, c'est lors d'une conférence de Nino Pirotta, donnée vers 1956, que j'entendis pour la première fois le terme de "stratification » appliqué à la musique ${ }^{1}$. La différence entre les trois strates du motet médiéval qu'il décrivit était manifeste, et j'ai tout de suite pensé que le terme de stratification convenait également aux pratiques musicales répandues en Asie du sud-est et ailleurs ${ }^{2}$. Le terme en question, comme la plupart des concepts de théorie musicale, se prêtait à être appliqué abusivement à la musique de la période classique, qui semblait remplir deux conditions 
préalables : d'une part, une densité relative propre à une strate donné (une mélodie typique de violon, par exemple, est plus dense, elle comprend plus de notes par mesure que celle du violoncelle ou de la contrebasse); et d'autre part le fait que cette densité puisse être considérée comme l'idiome de l'instrument en question, bien que la déviation par rapport à cette norme soit la règle plutôt que l'exception. C'est en effet dans la déviation de la densité idiomatique par rapport à la norme que réside la distinction essentielle.

4 La notion de stratification, qu'elle soit appliquée au gamelan javanais ou au motet médiéval, devrait se limiter à un ensemble constitué de strates à densité idiomatique variable. Jaap Kunst (1973) a attiré l'attention sur les contraintes pesant sur l'improvisation javanaise, dues en partie à l'idiome individuel de chaque instrument et de chaque voix ${ }^{3}$.

5 La polyphonie de la Renaissance obéit à une pratique modale qui comporte des implications harmoniques évidentes. La polyphonie baroque est organisée selon une pratique harmonique qui l'emporte sur les considérations modales. Quant à la polyphonie du gamelan javanais, elle est régie par le principe de la stratification idiomatique, tel qu'il vient d'être défini, et celui-ci est gouverné, à son tour, par les exigences de neuf niveaux de référence.

6 En résumé, les trois types de polyphonie - modale, tonale, et stratification polyphonique - se distinguent par leur base d'organisation. Avant de donner des exemples de pratiques musicales pertinentes en Asie du sud-est, je voudrais spécifier certains principes régissant la stratification polyphonique dans la musique du gamelan javanais.

7 J'ai déjà décrit le sens du «temps parfait » que les musiciens javanais semblent avoir développé ; par exemple, il y a de fortes chances qu'une pièce de gamelan jouée hier en 8'42" aura la même durée, à quelques secondes près, quand elle sera rejouée plus tard. Je crois que ce remarquable sens du temps absolu résulte de ce que j’ai appelé la « densité de saturation ", c'est-à-dire l'intégration de toutes les pulsations rapides de la "densité confortable » la plus rapide, quel que soit le tempo ${ }^{4}$. En d'autres termes : si, par exemple, le xylophone gambang joue huit notes pour chaque note de la mélodie principale, et que le tempo se ralentit sensiblement, l'instrumentiste double la densité de son jeu improvisé, de manière à émettre seize notes pour chaque note de la mélodie principale. Une fois que le nouveau tempo s'est installé, la densité de saturation parmi les lignes musicales les plus rapides devient la norme.

8 C'est dans la région centrale de Java que l'on rencontre la forme la plus complexe de stratification polyphonique. Selon la taille de l'ensemble, le nombre de strates peut varier entre quinze et vingt-cinq couches différentes d'activité mélodico-rythmique. En voici un exemple : le gong le plus grand possède la densité la plus faible, c'est-à-dire qu'à une de ses frappes correspondent seize frappes de la mélodie de base; les gongs plus petits résonnent une fois toutes les quatre pulsations; les instruments mélodiques sonnent à chaque pulsation, l'octave la plus aiguë de cette famille de saron émettant deux notes pour chaque pulsation; quelques instruments d'improvisation émettent quatre notes pour chaque pulsation, d'autres huit, d'autres encore seize, valeur qui peut atteindre trentedeux dans le tempo le plus lent, avec la possibilité idiomatique de redoublements ornementaux momentanés. L'instrument du meneur, le rebab, une vièle à pique grattée à deux cordes, possède une densité plus variable, notamment pour ce qui est du recours à l'ornementation (gregel) ; néanmoins, son idiome instrumental est distinct de tous les autres. Notons en passant que la mélodie de base de seize pulsations par rapport au gongan ne peut 
être que huit, ou alors de $32,64,128,256$. Toutes les formules mélodiques et rythmiques sont quadratiques, ce qui est un trait caractéristique de la stratification polyphonique dans toute l'Asie du sud-est ${ }^{5}$.

Fig. 1 : Vièle rabab et flûtes suling gambuh de l'ensemble de I Ketut Kantor, Batuan, Bali

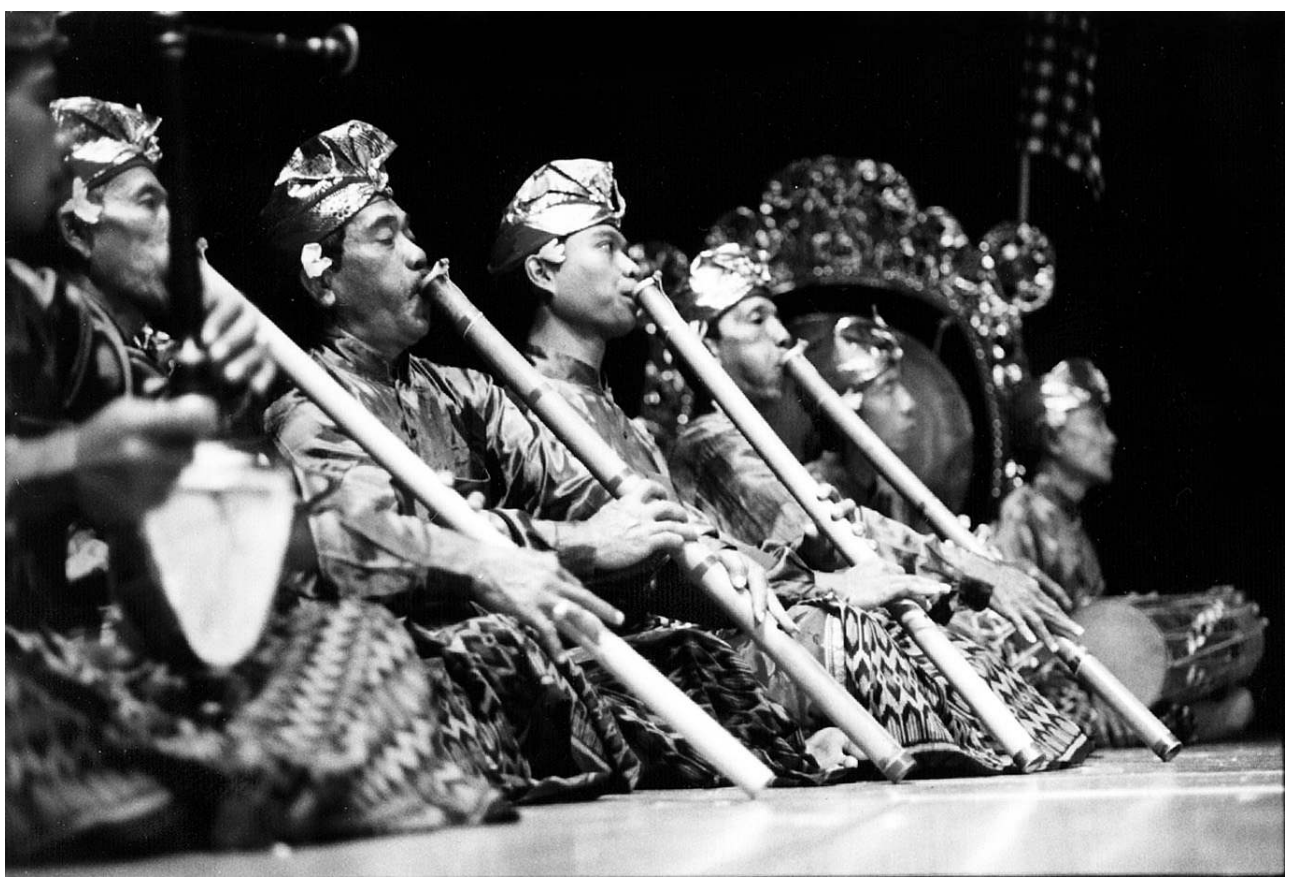

Photo : Jean-Elie Battista, 1993

9 C'est pourquoi l'improvisation de groupe, c'est-à-dire l'improvisation exécutée simultanément par la majorité des musiciens, se réalise dans toute la diversité des densités idiomatiques de l'ensemble. L'évolution mélodico-rythmique de l'improvisation collective est régie par ce que j'ai appelé ailleurs « les neuf niveaux ${ }^{6}$ que je me borne ici à énumérer simplement. Ces niveaux constituent une sorte de continuum allant du général au particulier :

- I. système d'accord ;

- II. mode ;

- III. colotomy ${ }^{7}$;

- IV. balungan, qui est une abstraction mélodique de :

- V. mélodie fixe ;

- VI. idiome instrumental-vocal ;

- VII. style local ;

- VIII. empathie de groupe ;

- IX. style personnel8.

10 En l'absence des contraintes imposées par ces neuf niveaux et par les idiomes individuels des instruments et des voix, il y aurait une sorte d'anarchie musicale, comme dans le cas des pétards chinois qu'on tire tous en même temps. En résumé, la pratique polyphonique dans le gamelan javanais est basée sur l'improvisation de groupe et organisée par la stratification idiomatique selon les neuf niveaux.

11 La stratification polyphonique d'autres ensembles de jeux de gongs d'Asie du sud-est fait état de différences de détail similaires, mais déterminées culturellement. A Bali, il n’y a 
presque pas d'improvisation. Il existe des exceptions à peine perceptibles dans des traditions comme le gamelan Semar Pegulingan, le gamelan Gong Gede et le gamelan Pelegongan : le trompong (jeu de gongs horizontaux couvrant plusieurs octaves) joué en soliste dans les deux premiers ensembles, et la paire de gender legong (métallophones couvrant plusieurs octaves) du troisième ensemble, qui sont tous trois des instruments meneurs de l'ensemble, exécutant des ornementations stéréotypées avec de légères variations. Dans l'ancien gamelan Gambuh, deux ou quatre suling gambuh (flûtes à embouchure terminale, longues d'un mètre) jouent à l'unisson - mises à part quelques variations minimes - des ornementations sur une mélodie principale sous-entendue ( pokok). Lorsque le rebab est présent dans l'un de ces ensembles, celui-ci exécute également une ornementation légèrement variable. A part ces ornements éphémères, aucun des ensembles mentionnés ne pratique l'improvisation. Dans le gamelan gong Kebyar moderne, dans le très ancien gamelan Angklung, dans le quatuor de métallophones gender wayang, dans certains ensembles très ancien ou, enfin, dans le style appelé kotekan (jeu rapide de parties imbriquées que connaissant la plupart des ensembles), il n'y a pas d'improvisation autre qu'une ornementation passagère introduite par l'instrument mélodique du meneur.

12 La riche polyphonie de ces ensembles est due à une forme de stratification idiomatique, qui peut englober quatre lignes ou plus dans le cas du quatuor gender wayang, voire un nombre de lignes qui est quatre ou cinq fois plus élevé, dans le cas des grands gamelan. La structure métrique de la musique est quadratique. Dans la plupart des cas, on retrouve en principe les neuf niveaux interdépendants évoqués précédemment comme autre facteur gouvernant la polyphonie non pas improvisée, mais fixe ${ }^{9}$.

Fig. 2 : Gongs du Gamelan du palais de Surakarta, Java

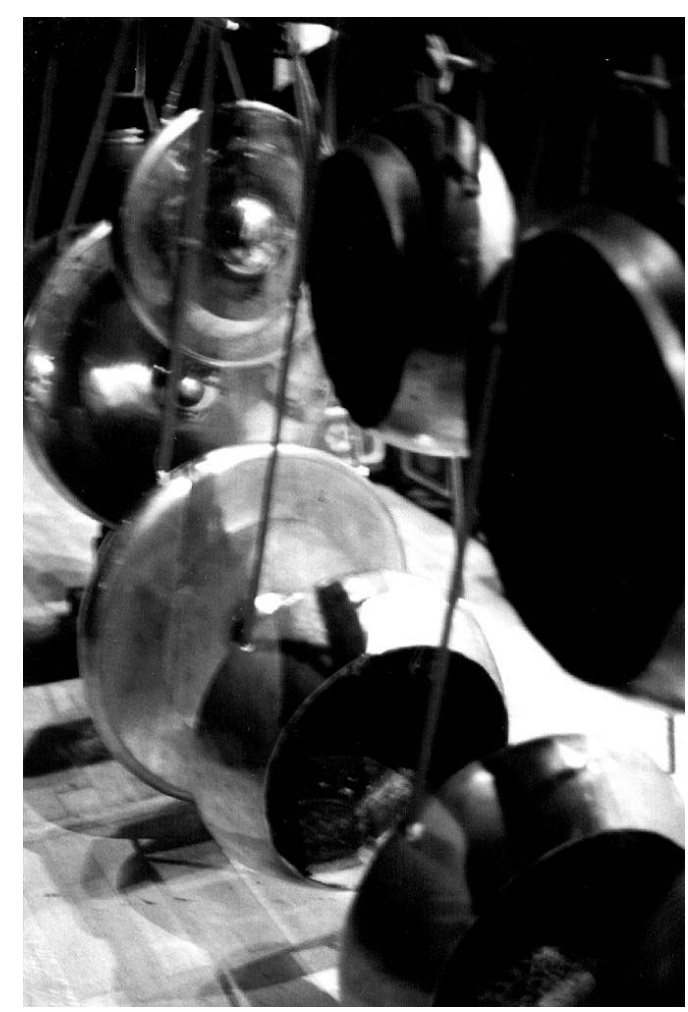

Cahiers d'ethnomusicologie, 6 | 2011 


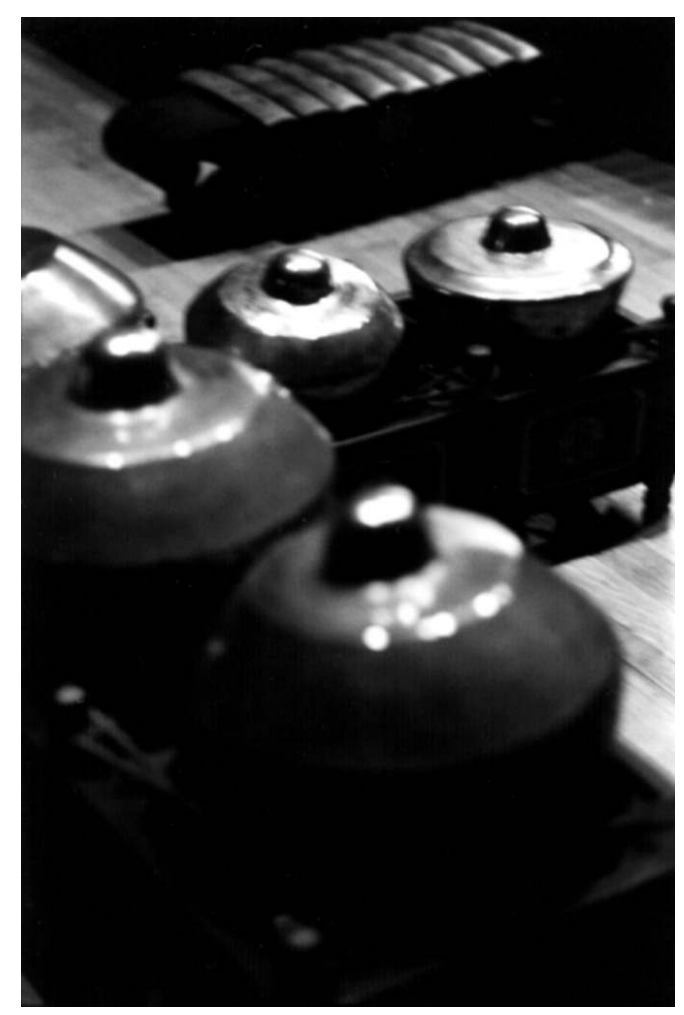

Photo : Jean-Elie Battista, 1993

13 A quelques exceptions près, les divers types de gamelan de Sunda (Java occidental) sont de taille nettement plus petite que les autres gamelan javanais. Leurs pratiques polyphoniques sont également organisées selon la stratification idiomatique et les neuf niveaux déjà mentionnés. Mais un trait unique à Sunda est le concept de surupan, qui implique un réseau de sous-modes fondé sur l'émission de « tons vocaux » par la voix, les instruments à cordes et la flûte, qui compliquent le mécanisme du niveau II, celui du mode, en guidant l'improvisation polyphonique à travers les strates idiomatiques de l'ensemble. Jaap Kunst a décrit ces dissonances intentionnelles entre un ton vocal et un «ton de bronze » proche (à hauteur fixe) comme le fondement d'une esthétique unique qui s'est développée à Sunda ${ }^{10}$.

14 A ce stade. il convient d'évoquer brièvement le petits groupes musicaux qui ne tombent habituellement pas sous la dénomination d'«ensembles de jeux de gongs », celle-ci se référant avant tout aux orchestres, de taille variable, où les gongs métalliques et les instruments à lames sont prédominants. Il existe dans toutes les cultures connaissant les jeux de gongs, de petits ensembles qui ne comprennent que peu d'instruments à gongs ou à lames en bronze (ou en fer ou en laiton), voire aucun. On comprend maintenant pourquoi le titre de cette contribution est «Stratification polyphonique dans la musique d'Asie du sud-est ». Ce ne sont pas seulement les ensembles à jeux de gongs et les motets médiévaux qui recourent au concept de stratification. La musique de Sunda, pour se limiter à un seul exemple parmi les nombreux cas rencontrés en Asie du Sud-Est, en fournit également une bonne illustration.

Un ensemble largement apprécié à Sunda est composé d'une voix chantant une belle poésie, d'une flûte à embouchure terminale (suling) qui est un peu plus grande et mélodiquement plus virtuose que sa contrepartie javanaise, et d'une grande cithare à 
cordes pincées (kecapi) sur laquelle le joueur produit un accompagnement rythmicomodal avec la main gauche et la mélodie principale avec la main droite. Le kecapi assume deux strates idiomatiques distinctes, le rebab la troisième, le suling la quatrième, et la voix le cinquième niveau idiomatique de la stratification polyphonique. L'organisation mélodico-rythmique de la musique à Sunda est quadratique.

Dans un livret accompagnant un disque de musique thaï ${ }^{11}$, David Morton s'est demandé si les orchestres pi-phat de Thaïlande n'étaient pas des "plantes de serre ", sous-entendant que leur existence contemporaine pourrait être le résultat d'une reconstruction, du moins partielle. Quoi qu'il en soit, ces ensembles thaï recourent, comme d'autres, à la stratification polyphonique, tout en étant gouvernés jusqu'à un certain point par les neuf niveaux. Dans le même texte, Morton présente les pratiques de ces ensembles comme des variations simultanées sur une mélodie principale, que distingue seulement leur densité idiomatique. Dans cette tradition, la variation est si stéréotypée qu'elle est loin de l'improvisation libre traditionnelle de Java - à l'exception notable du ranat ek, un type de xylophone, qui est devenu un véritable instrument de virtuose. La musique est quadratique.

Dans son excellente étude sur la musique traditionnelle du Cambodge, Giovanni Giurati nous révèle combien il est inexact et trompeur de rejeter le style de cette musique en n'y voyant qu'une simple imitation des pratiques thaï, comme l'a fait notamment David Morton. Contrairement à une " plante de serre ", l'ensemble pin péat du Cambodge assure une bonne dose d'improvisation de groupe. Bien qu'il s'agisse d'un ensemble plus petit que l'habituel gamelan javanais, son improvisation est, dans l'esprit comme dans la pratique, nettement plus proche de Java que des ensembles stéréotypés de Thaïlande (Giurati 1987). Au Cambodge également, ce sont l'équivalent des neuf niveaux de référence et la stratification polyphonique qui régissent l'improvisation. La musique est quadratique.

Le gamelan du théâtre Calonarang de Batuan, Bali

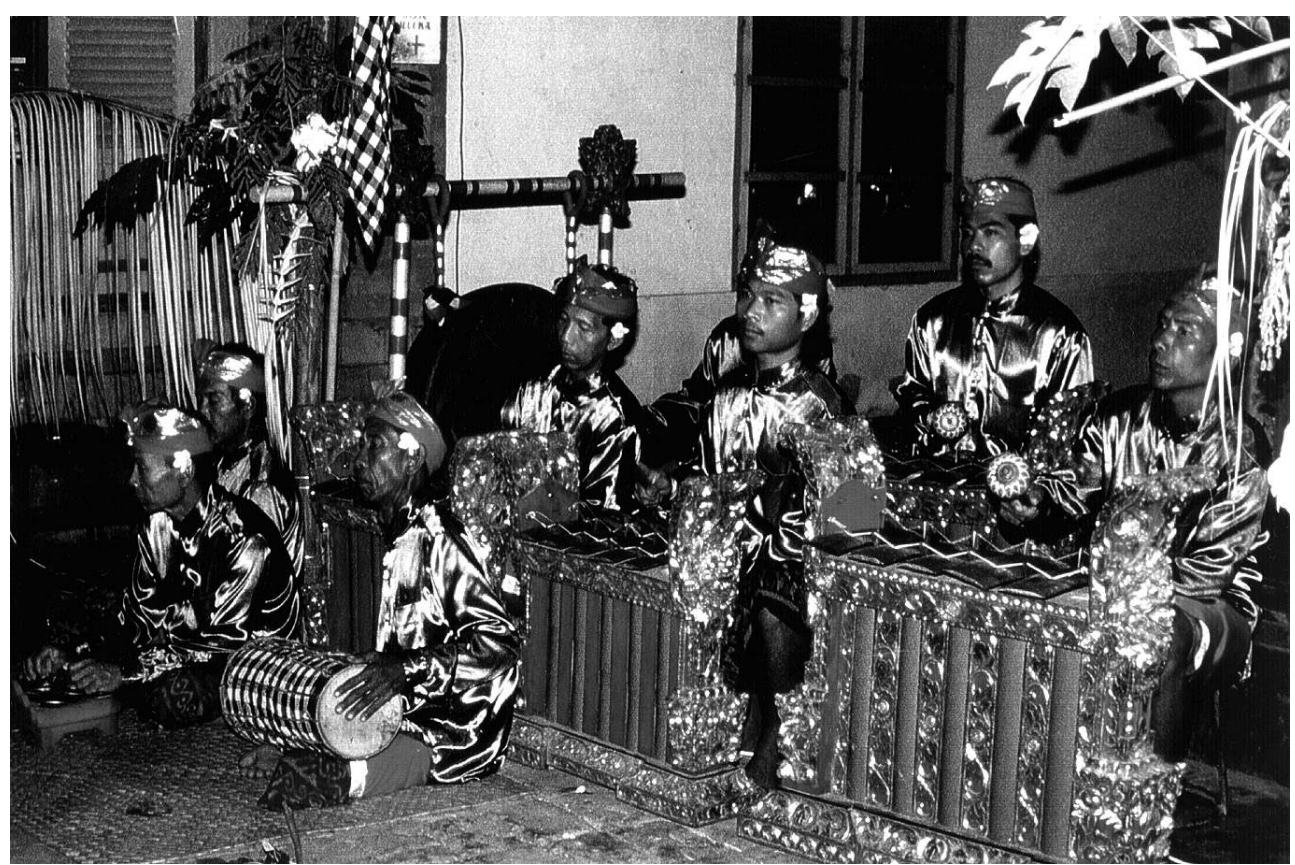


Photo : Thomas Kayser, 1993

18 Je connais moins bien les ensembles de Birmanie, qui sont également organisés selon le principe de la stratification polyphonique et gouvernés par des facteurs interdépendants comme les neuf niveaux ; mais à ma connaissance, il reste encore à en étudier les détails. A juger par l'écoute, il y a une plus grande liberté rythmique que dans les autres traditions d'Asie du sud-est. La musique est quadratique.

En résumé, la plupart des musiques traditionnelles d'Asie du sud-est, y compris tous les ensembles de jeux de gongs, obéissent tant à la stratification polyphonique qu'à neuf niveaux de référence interdépendants, dont le mode. Le mouvement polyphonique à travers ces strates idiomatiques peut être librement improvisé sur une mélodie fixe, ou rigidement prescrit, mise à part l'ornementation occasionnelle introduite par l'instrument meneur. La structure rythmique de toutes ces musiques est quadratique.

Je voudrais conclure en ouvrant une perspective sur les pratiques polyphoniques en Occident et en Orient. Dans la tradition européenne, la polyphonie a connu des variantes locales, régionales et nationales, mais celles-ci ont toujours été dominées par des pratiques modales ou harmoniques, voire, à présent, par des pratiques atonales et synthétiques. Voilà une évolution couvrant près d'un millénaire, depuis l'époque du motet médiéval à trois voix jusqu'au mouvement indépendant de «nuages sonores » le plus contemporain, quelle que soit la manière dont ces nuages sont articulées en théorie.

21 Un terme musical ne peut être compris que par référence à la pratique particulière qu'il signifie; en Occident, ces termes de référence ont changé selon les bases mêmes sur lesquelles la polyphonie a été fondée. Ce constat peut même valoir pour les extrêmes de l'avant-garde, qu'il s'agisse du mouvement de nuages sonores ou de la modification des partielles supérieures que j'ai entendue lors d'un concert de musique minimaliste avec un chœur produisant un bourdon de hauteur égale, mais ouvrant ou fermant plus ou moins la bouche. Voilà qui est également une forme de polyphonie, du moins si je saisis bien toutes les implications de ce terme, tel qu'il s'applique à la tradition européenne.

Mais même si le terme en question est pris dans son sens large, il ne couvre pas pour autant les pratiques d'Asie du sud-est que j'ai décrites dans ces lignes. Il y a, certes, des mouvements de voix multiples, mais dans la musique d'Asie du sud-est, c'est l'élément organisateur des densités idiomatiques stratifiées, interdépendantes et contrôlées par neuf niveaux de référence, qui est unique.

\section{BIBLIOGRAPHIE}

GIURIATI Giovanni, 1987, The Traditional Music of Cambodia. Baltimore : UMBC (thèse de doctorat inédite).

HOOD Ki Mantle, 1971, « Aspects of group improvisation in Javanese gamelan ». Music of Asia?

HOOD Ki Mantle, 1982, The Ethnomusicologist. Kent: Ohio State University Press. 
HOOD Ki Mantle, 1988, Paragon of the Roaring Sea, Book III: The Evolution of Javanese Gamelan.

Wilhelmshaven; Florian Noetzel Verlag.

HOOD Ki Mantle, 1990, « Balinese Gamelan Semar Pegulingan, the modal system ». Progress

Reports in Ethnomusicology 3(2):

KUNST Jaap, 1973, Music in Java. The Hague: Martinus Nijhoff (3e éd.).

PETERMAN Lewis E., 1989, « Regional variations in Balinese gender wayang music: a comparison and analysis of "gendhing rebong" ». Progress Reports in Ethnomusicology 2(7) : ?

\section{NOTES}

1. Lors d'un cours d'été à l'Université de Californie à Los Angeles.

2. Depuis ce jour-là, comme beaucoup d'autres, j'ai appliqué ce terme à des traditions comme la musique du Mexique, de l'Afrique et des orchestres de cour d'Extrême-Orient.

3. Dans la pratique vocale, la mélodie de la soliste - pesindenan - possède une densité idiomatique plus forte que la mélodie entonnée à l'unisson par le chœur masculin ; voir aussi Hood (1988 : 99-113, 268-74).

4. Cf. Hood (1982: 114-16) et Hood (1988: 201, 202-13).

5. Il arrive parfois que le bonang et le gambang du gamelan javanais exécutent des triolets. En 1957, un musicien m'indiqua à l'écoute que le tambourinaire émettait momentanément un rythme de valse qui se superposait à la structure quadratique du gamelan.

6. Pour plus de détails, voir Hood (1971; 1988 : 137-55).

7. Colotomy: terme adopté par Jaap Kunst pour décrire la structure en phrases des pièces de gamelan javanais.

8. Ce paradigme a notamment été utilisé par la suite dans l'étude de la musique hawaïenne (Elizabeth Tatar et al.), de la musique amérindienne (Charlotte Heth, Dale Olson), de la musique urbaine du Zaïre (Wa Mukuna Kazadi) et de la musicothérapie (Michel Rohrbacher).

9. Il convient de noter que les premières études sur les modes balinais ont été publiées tout à fait récemment; auparavant, l'existence d'une pratique modale dans la musique balinaise était controversée. Pour plus de détails, voir Hood (1990) et Peterman (1989).

10. Voir également l'Index du vol. II de Kunst (1973) ; les renvois de page figurent sous surupan (Kunst 1973 : 634).

11. Cf. le livret accompagnant le disque 33 tours The Traditional Music of Thailand (ier 7502) publié par l'Institut d'ethnomusicologie de l'Université de Californie à Los Angeles, en 1968.

\section{AUTEURS}

\section{KI MANTLE HOOD}

Ki Mantle Hood a obtenu son doctorat cum laude sous la direction de Jaap Kunst. En 1954, il a inauguré à l'Université de Californie de Los Angeles le premier programme universitaire en ethnomusicologie. En 1960, il a fondé le célèbre Institute of Ethnomusicology, qu'il a dirigé pendant quatorze ans et qui sera dissout peu après sa retraite, en 1974. Il est membre de 
l'Académie américaine des Arts et des Sciences. En 1966, le Gouvernement indonésien lui attribue le titre « Ki » (Le Vénérable) et, en 1992, il est élu au Darma Kusma ; il est le premier non-

Indonésien à accéder à ces honneurs. Depuis 1980, il est professeur (Senior Distinguished Professor) à l'Université de Maryland de Baltimore County (UMBC). Il est l'auteur de nombreux livres et articles. 